Pacific Journal of Mathematics

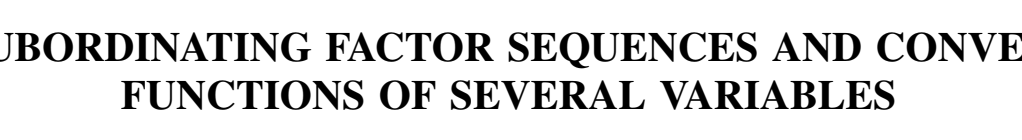




\title{
SUBORDINATING FACTOR SEQUENCES AND CONVEX FUNCTIONS OF SEVERAL VARIABLES
}

\author{
JAMES MiLLER
}

\begin{abstract}
In this paper we consider univalent holomorphic maps of $E^{n}$, the unit disk in $C^{n}$. We generalize Wilf's subordinating factor sequences to functions on $E^{n}$ and use this characterization to obtain a covering theorem and bounds for convex mappings in $C^{n}$.
\end{abstract}

1. Introduction. Let $K^{n}$ denote the class of functions $F$ which are holomorphic and univalent in $E^{n}=\left\{z=\left(z_{1}, \cdots, z_{n}\right): \operatorname{Max}_{1 \leqq i \leqq n}\left|z_{i}\right|<1\right\}$, maps $E^{n}$ onto a convex region in $C^{n}$, and satisfy $F(0)=0$ and the Jacobian $J$ of the mapping $F$ is nonsingular. Let $G$ and $H$ be holomorphic in $E^{n}$. If $G\left(E^{n}\right) \subset H\left(E^{n}\right)$, then $G$ is subordinate to $H(G \prec H)$. If $F=\left(F_{1}, \cdots, F_{n}\right) \in K^{n}$ then each $F_{i}$ has an expansion of the form

$$
F_{i}(Z)=\sum_{k=1}^{\infty} \sum_{\nu_{1}+\cdots+\nu_{n}=k} a_{\nu_{1} \cdots \nu_{n}}(i) z_{1}^{\nu_{1}} \cdots z_{n}^{\nu_{n}} .
$$

In this paper we characterize the sequences $\left\{c_{\nu_{1} \cdots \nu_{n}}(i)\right\}(i=1, \cdots, n)$ such that the mapping

$$
H=\left(H_{1}, \cdots, H_{n}\right)
$$

where

$$
H_{i}(Z)=\sum_{k=1}^{\infty} \sum_{\nu_{1}+\cdots+\nu_{n}=k} c_{\nu_{1} \cdots \nu_{n}}(i) a_{\nu_{1} \cdots \nu_{n}}(i) z^{\nu_{1}} \cdots z^{\nu_{n}}
$$

is subordinate to $F$, for all $F \in K^{n}$. Then we obtain a covering theorem and bounds for convex mappings.

For $n=1$, the class $K^{1}$ is the classical family of univalent functions $F(z)=\sum_{k=1}^{\infty} a_{k} z^{k}$ which maps the unit disk onto a convex domain. Wilf [4] has characterized the sequences $\left\{c_{k}\right\}$ (subordinating factor sequences) such that $h(z)=\sum c_{k} a_{k} z^{k}$ is subordinate to $f(z)=$ $\sum_{k=1}^{\infty} a_{k} z^{k}$ whenever $f \in K^{1}$. For $n>1$, Suffridge [3] has given the following characterization of the class $K^{n}$.

Theorem A. Suppose $F: E^{n} \rightarrow C^{n}$ is holomorphic, $F(0)=0$, and that $J$ is nonsingular for all $Z \in E^{n}$. Then $F$ is a univalent map of $E^{n}$ onto a convex domain if and only if there exists univalent mappings $f_{j} \in k^{1}(1 \leqq j \leqq n)$ such that $F(Z)=T\left(f_{1}\left(z_{1}\right), \cdots, f_{n}\left(z_{n}\right)\right)$ where $T$ is a nonsingular linear transformation. 
From Theorem A we see that if $F=\left(F_{1}, \cdots, F_{n}\right) \in K^{n}$ then

$$
F_{i}\left(z_{1}, \cdots, z_{n}\right)=\sum_{k=1}^{\infty}\left(a_{i 1}^{k} z_{1}^{k}+\cdots+a_{i n}^{k} z_{n}^{k}\right) .
$$

Thus we could represent $F \in K^{n}$ by the column vector

$$
F(Z)=\sum_{K=1}^{\infty} A_{k} Z^{k}
$$

where

$$
A_{k}=\left[\begin{array}{ccc}
a_{i 1}^{k} & \cdots & a_{\imath n}^{k} \\
\vdots & & \\
a_{n 1}^{k} & & a_{n n}^{k}
\end{array}\right] \quad Z^{k}=\left[\begin{array}{c}
z_{1}^{k} \\
\vdots \\
z_{n}^{k}
\end{array}\right]
$$

2. Subordinating factor sequences. An infinite sequence $\left\{C_{k}\right\}$ of $n \times n$ matrices of complex numbers will be called a subordinating factor sequence if for each $F(Z)=\sum A_{k} Z^{k} \in K^{n}$ we have $\sum C_{k} \odot A_{k} Z^{k} \prec$ $F(Z)$, where $C_{k} \odot A_{k}$ is the Hadamard product. If $C=\left(c_{i j}\right)$ and $A=\left(a_{i j}\right)$ then $C \odot A=\left(c_{i j} a_{i j}\right)$. Let $\mathscr{F}^{n}$ denote the collection of subordinating factor sequences.

THeOREM 1. If $\left\{C_{k}\right\} \in \mathscr{F}^{n}$, then for each $k$ the rows of $C_{k}=\left(c_{i j}^{k}\right)$ are identical, that is, for each $k(k=1,2, \cdots)$ and each $j(j=1, \cdots, n)$ we have $c_{1 j}^{k}=c_{2 j}^{k}=\cdots=c_{n j}^{k}$.

Proof. Let $\left\{C_{k}\right\} \in \mathscr{F}^{n}$. First consider $k=1$. Pick $\zeta=\left(\zeta_{1}, \cdots\right.$, $\left.\zeta_{n}\right) \in E^{n}$ where $\zeta_{i} \neq 0$ and if $c_{j j}^{1} \neq 0$ then $\zeta_{j}=1 / 2 e^{-i \alpha}$ with $\alpha=\arg c_{j j}^{1}$ if $c_{j j}^{1}=0$ then $\zeta_{j}=0$. Let $\delta=\left(c_{j i}^{1}-c_{i i}^{1}\right) \zeta_{i}$. If $\delta=0$, then $c_{j i}^{1}=c_{i i}^{1}$. If $\delta \neq 0$, let $M=1 / \delta$. Then define the mapping $F=\left(F_{1}, \cdots, F_{n}\right)$ where $F_{i}(Z)=M z_{i}, F_{j}(Z)=M z_{i}+z_{j}$, and $F_{k}(Z)=z_{k}$ when neither $k \neq i$ or $k \neq j$. The mapping $F$ is a convex univalent map by Theorem A. Thus since $\left\{C_{k}\right\} \in \mathscr{F}^{n}$ the mapping $H=\left(H_{1}, \cdots H_{n}\right)$, where $H_{i}(Z)=M c_{\imath \imath}^{1} z_{i}, H_{j}(Z)=M c_{j i}^{1} z_{i}+c_{j j}^{1} z_{j}$ and $H_{k}(Z)=c_{k k}^{1} z_{K}$ for $k \neq i$ or $k \neq j$, is subordinate to $F$. In particular, there is a $Z \in E^{n}$ such that $H(\zeta)=F(Z)$, which says

$$
M z_{i}=M c_{i i}^{1} \zeta_{i}
$$

and

$$
M z_{i}+z_{j}=M c_{j i}^{1} \zeta_{i}+c_{j j}^{1} \zeta_{j} .
$$

Solving for $z_{j}$ we obtain

$$
z_{j}=M\left(c_{j \imath}^{1}-c_{i i}^{1}\right) \zeta_{i}+c_{j j}^{1} \zeta_{j}=1+\frac{1}{2}\left|c_{i j}^{1}\right| \geqq 1 .
$$


This contradicts the fact that $|Z|<1$. Thus we have $\delta=0$ or $c_{1 j}^{1}=c_{2 j}^{1}=\cdots=c_{n j}^{1}$ for $j=1, \cdots, n$.

For $k>1$ we define the mapping $F=\left(F_{1}, \cdots, F_{n}\right)$ where

$$
F_{i}(Z)=M z_{i}+\frac{M z_{j}^{k}}{k^{2}}, F_{j}(Z)=M z_{\imath}+\frac{M z_{i}^{k}}{k^{2}}+z_{j}, \quad \text { and } \quad F_{k}(Z)=z_{k}
$$

for neither $k \neq i$ or $k \neq j$. Then the proof that $c_{1 j}^{k}=c_{2 j}^{k}=\cdots=c_{n_{j}}^{k}$ is similar to the proof for $k=1$.

From Theorem 1 we have that if $\left\{C_{k}\right\} \in \mathscr{F}^{n}$, then for each $k$ the rows of $C_{k}$ are indentical. For the $n \times n$ matrices $C_{k}$ we will use the notation

$$
C_{k}=\left[\begin{array}{ccc}
c_{1}^{k} \cdots & c_{n}^{k} \\
\vdots & & \\
c_{1}^{k} \cdots & c_{n}^{k}
\end{array}\right]=\left(c_{1}^{k}, \cdots, c_{n}^{k}\right)
$$

Using Theorem 1 we are now able to characterize class $\mathscr{F}^{n}$.

THEOREM 2. The following are equivalent:

(i ) $\left\{C_{K}\right\} \in \mathscr{F}^{n}$ where $C_{K}=\left(c_{1}^{k}, \cdots, c_{n}^{k}\right)$.

(ii) For each $j=1, \cdots, n$ we have

$$
\operatorname{Re}\left\{1+2 \sum_{k=1}^{\infty} c_{j}^{k} z_{j}^{k}\right\}>0 \text { for } \quad\left|z_{j}\right|<1
$$

(iii) For each $j=1, \cdots, n$ there is a nondecreasing function $\Psi_{j}$ on $[0,2 \pi]$ such that

$$
c_{j}^{k}=\frac{1}{2 \pi} \int_{0}^{2 \pi} e^{-i k \theta} d \Psi_{j}(\theta) \quad \text { and } \quad c_{j}^{\vartheta}=1 .
$$

Proof. The Herglotz's integral representation for positive harmonic functions proves that (ii) and (iii) are equivalent. Let $\left\{C_{k}\right\} \in$ $\mathscr{F}^{n}$, where $C_{k}=\left(c_{1}^{k}, \cdots, c_{n}^{k}\right)$. Let $f_{i}\left(z_{i}\right)=z_{i} /\left(1-z_{i}\right)$. Then by Theorem A the mapping $F$ is in $K^{n}$. We may write

$$
F(Z)=\sum_{k=1}^{\infty} A_{k} Z^{k}
$$

where $A_{k}=\left(a_{\imath j}^{k}\right)$ and $a_{j i}^{k}=0$ if $i \neq j$ and $a_{\imath i}^{k}=1$ then the mapping

$$
H(Z)=\sum_{k=1}^{\infty} C_{k} \odot A_{k} Z^{k}
$$

is subordinate to $F$. The mapping $H$ has components $H_{i}(Z)=$ $\sum_{k=1}^{\infty} c_{i}^{k} z_{i}^{k}$. Since $H \prec F$ we have that $H_{i}\left(F_{i}\right) \subset f_{i}\left(E_{i}\right)$ or $\operatorname{Re}\left\{H_{i}\left(E_{i}\right)\right\} \geqq$ $-1 / 2$ where $E_{i}=\left\{z_{i}:\left|z_{i}\right|<1\right\}$. Thus $\operatorname{Re}\left\{\sum_{k=1}^{\infty} c_{i}^{k} z_{i}^{k}\right\}>-1 / 2$ for $i=$ 
$1, \cdots, n$, Now suppose (iii) holds. Let $F \in K^{n}$. Then by Theorem A there exists a nonsingular matrix $T$ and functions $f_{1}, \cdots, f_{n} \in K^{1}$, where $f_{i}\left(z_{i}\right)=\sum_{k=1}^{\infty} a_{k}(i) z_{i}^{k}$, such that

$$
F(Z)=T\left[\begin{array}{c}
f_{1}\left(z_{1}\right) \\
\vdots \\
f_{n}\left(z_{n}\right)
\end{array}\right]
$$

where $F$ is a column vector. Then

$$
\begin{aligned}
H(Z) & =\sum C_{k} \odot A_{k} z^{k}=T\left[\begin{array}{l}
\sum_{k=1}^{\infty} c_{1}^{k} a_{k}(1) z_{1}^{k} \\
\vdots \\
\sum_{k=1}^{\infty} c_{n}^{k} a_{k}(n) z_{n}^{k}
\end{array}\right] \\
& =T\left[\begin{array}{l}
\sum_{k=1}^{\infty} \frac{1}{2 \pi} \int_{0}^{2 \pi} e^{i k \phi} d \Psi_{1}(\phi) a_{k}(1) z_{1}^{k} \\
\vdots \\
\sum_{k=1}^{\infty} \frac{1}{2 \pi} \int_{0}^{2 \pi} e^{i k \phi} d \psi_{n}(\phi) a_{k}(n) z_{n}^{k}
\end{array}\right] \\
= & T\left[\begin{array}{l}
\frac{1}{2 \pi} \int_{0}^{2 \pi} \sum_{k=1}^{\infty} a_{k}(1) r_{1}^{k} e^{i j\left(\theta_{1}+\phi\right)} d \Psi_{1}(\phi) \\
\vdots \\
\frac{1}{2} \int_{0}^{2 \pi} \sum_{k=1}^{\infty} a_{k}(n) r_{n}^{k} e^{i k\left(\theta_{n}+\phi\right)} d \Psi_{n}(\phi)
\end{array}\right] \\
= & T\left[\begin{array}{l}
\frac{1}{2 \pi} \int_{0}^{2 \pi} f_{1}\left(r_{1} e^{i\left(\theta_{1}+\psi r\right)}\right) d \Psi_{n}(\phi) \\
\vdots \\
\frac{1}{2 \pi} \int_{0}^{2 \pi} f_{n}\left(r_{n} e^{i\left(\theta_{n}+\phi\right)}\right) d \Psi_{n}(\phi)
\end{array}\right]
\end{aligned}
$$

where $z_{j}=r_{j} e^{i \theta_{j}}$. Since each integral in the left hand side is the centroid of a nonnegative mass distribution of total mass one on a convex curve, the value of each integral must lie inside its convex curve. Further since $T$ is a nonsingular linear transformation $H(Z)$ lies inside the image of the polydisk of radius $\left(r_{1}, \cdots, r_{n}\right)$. (A polydisk or radius $\left(r_{1}, \cdots, r_{n}\right)$ is the set $\left\{\left(z_{1}, \cdots, z_{n}\right):\left|z_{i}\right| \leqq r_{i}\right.$ for $i=1, \cdots, n\}$.) Thus $H \prec F$.

3. Convex mappings in $C^{n}$. We now apply Theorem 2 to obtain some results for mapping in $K^{n}$.

Corollary 1. For $n>1$ let $G \in K^{n}$, where $G(Z)=\sum B_{k} Z^{k}$. 
Then the mapping

$$
G_{F}^{*}(Z)=\sum B_{k} \odot A_{k} Z^{k},
$$

where $F(Z)=\sum A_{k} Z^{k} \in K^{n}$, is not subordinate to $F$ for all $F \in K^{n}$.

Proof. If $G_{F}^{*} \prec F$ for all $F \in K^{n}$, then the sequence $\left\{B_{k}\right\}$ belongs to $\mathscr{F}^{n}$. This says that the rows of each $B_{k}$ are indentical by Theorem 1. Hence the Jacobian of $G$ will be identically zero. Thus $G_{F}^{*}$ is not subordinate to $F$ for all $F \in K^{n}$.

Let $T=\left(t_{i j}\right)$ be a $n \times n$ nonsingular matrix. Let $K$ be the functions $f \in K^{1}$ where $f^{\prime}(0)=1$. Let $K T$ denote the subclass of $K^{n}$ which is defined by $F \in K T$ if and only if there exist functions $f_{i} \in K(i=1,2, \cdots, n)$ such that

$$
F(Z)=T\left(\begin{array}{c}
f_{1}\left(z_{1}\right) \\
\vdots \\
f_{n}\left(z_{n}\right)
\end{array}\right)
$$

where $F$ is represented as a column vector.

CoRollary 2. The image of $E^{n}$ under a mapping $F \in K T$ contains the polydisk $|w|<1 / 2\left(\sum_{j=1}^{n}\left|t_{i j}\right|, \cdots, \sum_{j=1}^{n}\left|t_{n j}\right|\right)$. The radius is sharp.

Proof. Since the sequence $\left\{C_{k}\right\}$ where $C_{1}=(1 / 2,1 / 2, \cdots, 1 / 2)$ and $C_{k}=(0, \cdots, 0)$ for $k \geqq 2$, belongs to $\mathscr{F}^{n}$, we see that the image of $E^{n}$ under a mapping $F \in K T$ contains $|W|<1 / 2\left(\sum_{j=1}^{n}\left|t_{1 j}\right|, \cdots\right.$, $\left.\sum_{j=1}^{n}\left|t_{n k}\right|\right)$. The sharpness follows by using the function

$$
F(Z)=T\left[\begin{array}{c}
\frac{z_{1}}{1-z_{1}} \\
\vdots \\
\frac{z_{n}}{1-z_{n}}
\end{array}\right]
$$

Ruscheweyh and Sheil-Small [2] have proven Pólya and Schoenberg's [1] conjecture that if $f(z)=\sum_{k=1}^{\infty} a_{k} z^{k}$ and $g(z)=\sum b_{k} z^{k}$ are elements of $K^{1}$ then so is the function $h(z)=\sum a_{k} b_{k} z^{k}$. In general for $K^{n}$ this is not true as shown by the example $F(Z)=\left(\begin{array}{l}z_{1}-z_{2} \\ z_{1}+z_{2}\end{array}\right)=$ $G(Z)$. However, we do have the following Pólya and Schoenberg tpye of theorem.

THEOREM 3. Let $T_{1}=\left(p_{i j}\right)$ and $T_{2}=\left(q_{\imath j}\right)$ be $n \times n$ nonsingular matrices such that $T=T_{1} \odot T_{2}=\left(p_{i j} q_{i j}\right)$ is nonsingular. If $F(Z)=$ 
$\sum_{k=1}^{\infty} A_{k} Z^{k} \in K T_{1}$ and $G(Z)=\sum_{k=1}^{\infty} B_{k} Z^{k} \in K T_{2}$, then $H(Z)=\sum_{k=1}^{\infty} A_{k}$ $B_{k} Z^{k}$ belongs to $K T$.

Proof. Let $F \in K T_{1}$ and $G \in K T_{2}$. Then there exists functions $f_{i}, g_{i} \in K(i=1, \cdots, n)$ such that

$$
F(Z)=T_{1}\left[\begin{array}{c}
f_{1}\left(z_{1}\right) \\
\vdots \\
f_{n}\left(z_{n}\right)
\end{array}\right]
$$

and

$$
G(Z)=T_{2}\left[\begin{array}{c}
g_{1}\left(z_{1}\right) \\
\vdots \\
g_{n}(z)_{n}
\end{array}\right]
$$

The mapping $H(Z)=\sum_{k=1}^{\infty} A_{k} \odot B_{k} z^{k}$ may be written as

$$
H(Z)=T\left(\begin{array}{c}
z_{1}+\sum_{k=1}^{\infty} a_{k}(1) b_{k}(1) z_{1}^{k} \\
\vdots \\
z_{n}+\sum_{k=2}^{\infty} a_{k}(n) b_{k}(n) z_{k}^{n}
\end{array}\right) .
$$

Thus $H \in K T$ since $z_{i}+\sum a_{k}(i) b_{k}(i) z_{i}^{k}$ belongs to $K$ for each $i$ [2].

4. Bounds on Mapping in $K_{n}$. Let $F \in K^{n}$. Then by Suffridge's representation of mappings in $K^{n}$ (Theorem A), there exist an $n \times n$ nonsingular matrix $T=\left(t_{i j}\right)$ and functions $f_{i}\left(z_{i}\right)=\sum_{k=1}^{\infty} a_{k}(i) z_{i}^{k}(i=1, \cdots, n)$ in $K^{1}$ with $f_{1}^{\prime}(0)=1$ such that

$$
F(Z)=T\left(\begin{array}{c}
f_{1}\left(z_{1}\right) \\
\vdots \\
f_{n}\left(z_{n}\right)
\end{array}\right) .
$$

Then

$$
A_{k}=\left(a_{i j}\right)=T\left(\begin{array}{c}
a_{k}(1) \\
\vdots \\
a_{k}(n)
\end{array}\right)
$$

where $F(z)=\sum_{k=1}^{\infty} A_{k} Z^{k}$. Since

$$
\left|a_{k}(i)\right|<1 \text { and } \frac{\left|z_{i}\right|}{1+\left|z_{i}\right|}<\left|f_{i}\left(z_{i}\right)\right|<\frac{\left|z_{i}\right|}{1-\left|z_{i}\right|},
$$

we have the following theorem. 
TheOREM 4. Let $F(z)=\sum_{k=1}^{\infty} A_{k} Z^{k}$ belongs to $K^{n}$. Let $T$ be an $n \times n$ nonsingular matrix and let $f_{1}, \cdots, f_{n} \in K^{1}$ such that

$$
F(Z)=T\left(\begin{array}{c}
f_{1}\left(z_{1}\right) \\
\vdots \\
f_{n}\left(z_{n}\right)
\end{array}\right) .
$$

Then

$$
\left|a_{i j}^{k}\right|<\left|t_{i j}\right|
$$

for each $k$, $i$, and $j$, where $A_{k}=\left(a_{\imath j}^{k}\right)$. Let $F=\left(F_{1}, \cdots, F_{n}\right)$. Then

$$
\sum_{j=1}^{n}\left|t_{i j}\right| \frac{\left|z_{j}\right|}{1+\left|z_{j}\right|} \leqq\left|F_{\imath}(Z)\right|<\sum_{j=1}^{n}\left|t_{i j}\right| \frac{\left|z_{j}\right|}{1-\left|z_{j}\right|} .
$$

Both inequality are sharp.

\section{REFERENCES}

1. G. Pólya and I. J. Schoenberg, Remarks on de la Vallie Poussin means and convex conformal maps of the unit circle, Pacific J. Math., 8 (1958), 295-334.

2. St. Ruscheweyh and T. Sheil-Small, Hadamard products of schlicht functions and the Polya-Schoenberg conjecture, Comment. Math. Helv., 48 (1973), 119-135.

3. T. J. Suffridge, The principle of subordination applied to functions of several variables, Pacific J. Math., 33 (1970), 241-248.

4. H. S. Wilf, Subordinating factor sequences for convex maps of the unit circle, Proc. Amer. Math. Soc., 12 (1961), 689-693.

Received April 30, 1975.

WEST VIRGINIA UNIVERSITY 



\section{PACIFIC JOURNAL OF MATHEMATICS}

\section{EDITORS}

RICHARD ARENS (Managing Editor) University of California

Los Angeles, California 90024

R. A. Beaumont

University of Washington

Seattle, Washington 98105
J. DUGUNDJI

Department of Mathematics University of Southern Californı

Los Angeles, California 90007

D. Gilbarg and J. Milgram

Stanford University

Stanford, California 94305

\section{ASSOCIATE EDITORS}
E. F. BECKENBACH
B. H. NeumanN
F. WOLF
K. YosHIDA

\section{SUPPORTING INSTITUTIONS}

UNIVERSITY OF BRITISH COLUMBIA

CALIFORNIA INSTITUTE OF TECHNOLOGY

UNIVERSITY OF CALIFORNIA

MONTANA STATE UNIVERSITY

UNIVERSITY OF NEVADA

NEW MEXICO STATE UNIVERSITY

OREGON STATE UNIVERSITY

UNIVERSITY OF OREGON

OSAKA UNIVERSITY
UNIVERSITY OF SOUTHERN CALIFORNIA

STANFORD UNIVERSITY

UNIVERSITY OF TOKYO

UNIVERSITY OF UTAH

WASHINGTON STATE UNIVERSITY

UNIVERSITY OF WASHINGTON

* * * *

AMERICAN MATHEMATICAL SOCIETY

NAVAL WEAPONS CENTER 


\section{Pacific Journal of Mathematics \\ Vol. 65, No. $1 \quad$ September, 1976}

David Lee Armacost, Compactly cogenerated LCA groups ............. 1

Sun Man Chang, On continuous image averaging of probability measures ...... 13

J. Chidambaraswamy, Generalized Dedekind $\psi$-functions with respect to a

polynomial. II................................... 19

Freddy Delbaen, The Dunford-Pettis property for certain uniform algebras ..... 29

Robert Benjamin Feinberg, Faithful distributive modules over incidence

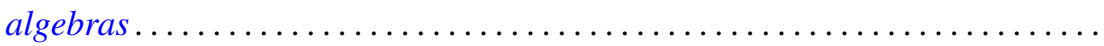

Paul Froeschl, Chained rings . . . . . . . . . . . . . . . . . . . .

John Brady Garnett and Anthony G. O'Farrell, Sobolev approximation by a sum

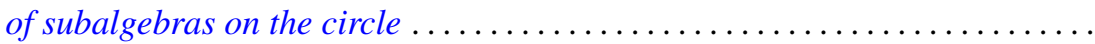

Hugh M. Hilden, José M. Montesinos and Thomas Lusk Thickstun, Closed

oriented 3-manifolds as 3-fold branched coverings of $S^{3}$ of special type.....

Atsushi Inoue, On a class of unbounded operator algebras ................

Peter Kleinschmidt, On facets with non-arbitrary shapes.

Narendrakumar Ramanlal Ladhawala, Absolute summability of Walsh-Fourier

series

Howard Wilson Lambert, Links which are unknottable by maps . . . . . . . . . . .

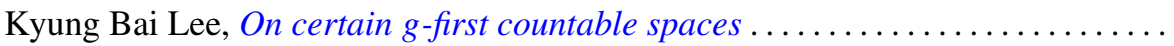

Richard Ira Loebl, A Hahn decomposition for linear maps .................

Moshe Marcus and Victor Julius Mizel, A characterization of functionals on $W_{1}^{p}$ possessing autonomous kernels. I . .

James Miller, Subordinating factor sequences and convex functions of several

variables.

Keith Pierce, Amalgamated sums of abelian l-groups ...

Jonathan Rosenberg, The $C^{*}$-algebras of some real and $p$-adic solvable

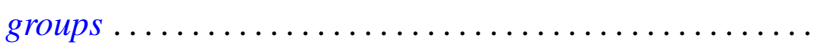

Hugo Rossi and Michele Vergne, Group representations on Hilbert spaces defined

in terms of $\partial_{b}$-cohomology on the Silov boundary of a Siegel domain . .

Mary Elizabeth Schaps, Nonsingular deformations of a determinantal

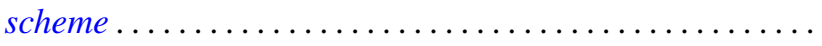

S. R. Singh, Some convergence properties of the Bubnov-Galerkin method...

Peggy Strait, Level crossing probabilities for a multi-parameter Brownian

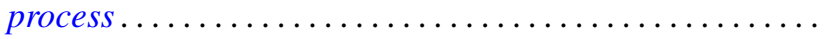

Robert M. Tardiff, Topologies for probabilistic metric spaces .

Benjamin Baxter Wells, Jr., Rearrangements of functions on the ring of integers of

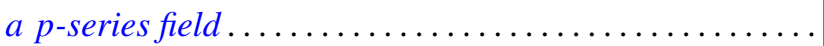

Robert Francis Wheeler, Well-behaved and totally bounded approximate identities for $C_{0}(X)$.

Delores Arletta Williams, Gauss sums and integral quadratic forms over local

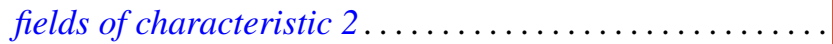

John Yuan, On the construction of one-parameter semigroups in topological 